\title{
An update on available pain medications
}

\author{
Elzbieta Osuch, ${ }^{1}$ Andre Marais ${ }^{2}$ \\ 'Department of Pharmacology \& Therapeutics, School of Medicine, Sefako Makghato Health Sciences University, South Africa \\ ${ }^{2}$ Department of Pharmacology, School of Medicine, Faculty of Health Sciences, University of Pretoria, South Africa \\ *Corresponding author, email:dramarais@gmail.com / andre.marais@up.ac.za
}

\begin{abstract}
Pain is a complex interaction of sensory, emotional and behavioural factors. Chronic pain is among the most common reasons for seeking medical attention. In contrast to acute pain, where the goal is primary pain relief, the effective management of chronic pain is complex and not fully understood. Multimodal pain therapy is often required to treat chronic or persistent pain syndromes. These include pharmacological agents, physical interventions, behavioural changes and surgical approaches. Pharmacological interventions are the most widely used therapeutic options to treat acute and persistent pain.
\end{abstract}

Keywords: pain, paracetamol, NSAIDs, COX-2 inhibitors, opioids, antidepressants, anticonvulsants

\section{Introduction}

Pain is often a major symptom in various medical conditions and is reported in nearly $20 \%-50 \%$ of patients seen in primary care. ${ }^{1,2}$ The International Association for the Study of Pain defines pain as "unpleasant sensory and emotional experience associated with actual or potential tissue damage, or described in terms of such damage".3 The global prevalence of chronic pain is estimated to be nearly $40 \%$, and is therefore considered to pose major medical and social complications, affecting the quality of life of patients, their families and society. ${ }^{1-4}$ In the United States, chronic pain affects more than 100 million people annually. This accounts for $20 \%$ of outpatient visits and $12 \%$ of all prescriptions being issued. 4,5 Pain-related expenditures exceed those for cancer, heart disease, and diabetes combined. ${ }^{6}$ There are few studies documenting the prevalence of chronic pain in South Africa with a reported prevalence of $42.3 \%$ amongst people living in rural communities and a similarly high prevalence of $41 \%$ in a more urban population in South West Tshwane. Pain had a severe impact on sleep quality in $39.2 \%$ patients, walking ability (37.4\%), routine housework $(33.8 \%), \operatorname{mood}(20.1 \%)$, interpersonal relationships (15.3\%) and enjoyment of life (16.3\%). ${ }^{7-9}$

\section{Classification of pain}

Pain can be classified according to the time course (acute, chronic), neurophysiological mechanism (nociceptive, neuropathic, inflammatory), intensity (mild-moderate-severe; 0-10 numeric pain rating scale), etiology (cancer, fibromyalgia, migraine, others) or type of tissue involved (skin, muscles, viscera, joints, tendons, bones).

Acute pain is defined as a pain of sudden onset, is short lasting, is usually the result of a clearly defined cause such as an injury, and resolves with the healing of its underlying cause. Chronic pain generally refers to intractable pain that exists for more than 3 months and does not resolve on treatment. ${ }^{10}$ Chronic pain is usually associated with an underlying condition. Acute pain when neglected can become chronic. ${ }^{11}$

Neurophysiological mechanism of pain has been categorised as nociceptive and non-nociceptive pain. Nociceptive pain is subdivided into somatic and visceral pain. ${ }^{3}$ Somatic pain results from excitation and sensitisation of nociceptors in tissues, such as skin, bone, peripheral soft tissue, joints, and muscles. Stimulation of these receptors usually produces sharp or dull well localised pain. Burning can occur if the skin or subcutaneous tissues are involved. Visceral pain receptors are located in most viscera and the surrounding connective tissue. Visceral pain is usually diffuse, poorly localised, often referred to other locations, and is often accompanied by nausea and vomiting.

Non-nociceptive pain can be sub-divided into neuropathic (either peripheral, including post-herpetic neuralgia, diabetic neuropathy; or central, including post-stroke pain or multiple sclerosis), inflammatory (eg, inflammatory arthropathies, infection), musculoskeletal (eg, back pain, myofascial pain syndrome), mechanical/compressive pain (eg, renal calculi, visceral pain from expanding tumor masses) and idiopathic pain/ psychogenic pain (myofascial pain syndrome and somatization pain disorder). Neuropathic pain can result from injury to neural structures within the peripheral or central nervous system. It is often described as: burning, shooting, stabbing, numbness or tingling. Therefore, many pain syndromes are often multifactorial and combinations of different types of pain exist. ${ }^{12}$

\section{Pain intensity scales}

Most measures of pain are based on self-report. Pain scales are reliable only when comparing the intensity of one patient's pain 
at different times. A number of different measurements for pain intensity can be implemented like: verbal scale, numerical rating scale (NRS), a visual analog scale (VAS), but no scale is clearly better than another. ${ }^{13-15}$

\section{Pathophysiology}

Acute pain, results from activation of peripheral nociceptors (A delta and $C$ sensory nerve fibres) due to the release of vasoactive peptides (eg, calcitonin gene-related protein, substance $P$, neurokinin $A$ ) and other mediators like proinflammatory cytokines, chemokines, vasoactive amines, prostaglandin $E_{2}$, serotonin, bradykinin and epinephrine. ${ }^{16}$ The activation of nociceptors transduce noxious stimuli into electrochemical impulses, which are transmitted to the spinal cord at the dorsal root ganglia (synapse in the dorsal horn where they cross to the other side) and then travel up the lateral columns to the thalamus and to the cerebral cortex. Repetitive stimulation from a prolonged painful condition can sensitise neurons in the dorsal horn of the spinal cord (wind-up phenomenon, less pain causing symptoms). Peripheral nerves and nerves at other levels of the CNS may also be sensitised, producing long-term synaptic remodeling in cortical fields that maintain exaggerated pain perception. The pain signal is modulated in segmental and descending pathways by many neurochemical mediators, including endorphins (eg, enkephalin) and monoamines (eg, serotonin, noradrenalin).

Persistent activation or aberrant somatosensory processing of sensory fibers causes chronic pain. The severity of tissue injury does not always predict the severity of chronic or acute pain. Psychologic factors (depression, anxiety) can modulate pain intensity to a highly variable degree. ${ }^{17}$

\section{Pharmacological management of pain}

A comprehensive pain evaluation is essential to developing an effective plan for treatment. In particular, neuropathic pain should be distinguished from nociceptive pain. The quality of pain, onset, location, duration, radiation, impact, severity, temporal characteristics, changes since onset, constancy or intermittency, characteristics of any breakthrough, pain exacerbating/triggering factors, palliative/relieving factors and associated symptoms should be determined. An appropriate history and physical examination are vital to a proper evaluation. Laboratory studies, imaging, and other diagnostic testing may be appropriate in selected situations. An approach using combinations of drugs may result in improved analgesia and fewer side effects, however, treatment response may differ between individuals, and not one approach is appropriate for all patients. Comorbidities should be evaluated and treated, in particular, major depression. ${ }^{18}$

The major pharmacological agents used in the management of pain include opioids (morphine and derivatives), and nonopioid analgesics (paracetamol, nonsteroidal anti-inflammatory drugs (NSAIDs) and selective cyclooxygenase type 2 inhibitors (COX-2 Inhibitors). Other entities such as antidepressants (tricyclics, serotonin-norepinephrine reuptake inhibitors), $a_{2}$ calcium channel ligands (gabapentin, pregabalin, and other anticonvulsants), muscle relaxants, N-methyl-d-aspartate (NMDA) receptor antagonists, and topical analgesic agents are often used as adjunct therapy. ${ }^{19}$ The effective management of pain requires a thorough understanding of the different pain mechanisms including the mechanistic action of the drugs used to manage this debilitating condition. The World Health Organization recommends a "ladder strategy" in the treatment of pain. Step 1 includes the use of non-opioid analgesics for mild pain. Step 2 requires adding a moderate opioid agonist to the non-opioids for a short duration. Step 3 recommends the continuous use of a high potency opioid for severe and persistent pain. The adjuvants can be added in all steps of treatment. ${ }^{20}$

The pharmacologic approach to nociceptive pain primarily involves non-narcotic and opioid analgesia. Paracetamol is recommended as a first-line therapy but is less effective than NSAIDs. ${ }^{21}$ NSAIDS may be used as alternative first line options for mild to moderate chronic pain.22 Chronic opioid therapy should only be considered in patients who are at low risk for substance abuse, and who experience persistent pain despite trials of non-opioid analgesics and antidepressants. Although opioid analgesics seem to have a significant benefit in acute pain conditions, their effectiveness in chronic use remains uncertain with an added risk of overdose resulting from dosage increases..$^{23}$

For most patients, the initial treatment of neuropathic pain involves either antidepressants or calcium channel alpha 2-delta ligands (gabapentin and pregabalin), with adjunctive topical therapy (eg, topical lidocaine). ${ }^{24}$ Serotonin-noradrenaline uptake inhibitors (SNRIs) are identified as either first or secondline agents, although they may be preferred to tricyclic antidepressants. Opioids are considered a second-line option, but may be used earlier in patients with severe intractable pain, frequent exacerbations, or neuropathic cancer pain. Combination therapy is often required, since less than half of patients with neuropathic pain will respond to a single agent. In the treatment of trigeminal neuralgia, carbamazepine or oxcarbazepine are the drugs of choice. ${ }^{25}$

\section{Nonsteroidal anti-inflammatory drugs (NSAIDs)}

NSAIDs are indicated for mild to moderate pain, particularly of inflammatory origin. The analgesic effect of NSAIDs is derived from the inhibitory action on the enzyme cyclooxygenase (COX-1 and COX-2). ${ }^{26}$ Short-to-moderate-acting NSAIDs (naproxen, ibuprofen) are preferred for most patients. Other available NSAIDS include diclofenac, indomethacin, ketorolac, ketoprofen, piroxicam, mefanamic acid and lornoxicam. Concurrent administration of two or more NSAIDs should be avoided since toxicity may be increased. In the absence of a favourable analgesic response, combination with nonopioid- or opioid-like drugs may be considered. NSAIDS have significant inter-individual differences regarding tolerability, pharmacokinetic profile and side effects among patients. All NSAIDs are associated with adverse gastrointestinal (dyspepsia, ulceration, bleeding), renal (interstitial nephritis, tubular necrosis, reversible renal insufficiency) dermatological, hepatic, haematological, immunological and neurological side effects. These are mostly dependent on hydration status, dosage and 
age of the patient. ${ }^{27}$ Alcohol may potentiate gastrointestinal side effects and administration with food is advised. Proton pump inhibitors (PPIs), misoprostol, or histamine type 2 receptor antagonists may be administered concurrently as gastrointestinal protective measures.

Non-selective NSAIDs reversibly inhibit platelet functioning and can alter the cardio-protective effects of aspirin, therefore their regular use should be avoided if possible in patients taking low dose aspirin or suffering from platelet dysfunction. ${ }^{28}$ Naproxen is the preferred nonselective agent in these patients, however, its use should be limited to the lowest effective dose for the shortest time period. In addition, NSAIDs have been associated with an increased risk of other adverse cardiovascular thrombotic events such as myocardial infarction and stroke. ${ }^{29}$ They are therefore contraindicated in the management of perioperative pain following coronary artery bypass graft surgery, unstable angina, and myocardial infarct. Caution needs to be exercised in patients with uncontrolled hypertension, heart failure, renal insufficiency, aspirin-sensitive asthma and individuals on concomitant anticoagulant treatment, lithium therapy, loop diuretics, glucocorticosteriods and ACEI. ${ }^{30}$

\section{Selective and specific cyclo-oxygenase-2 inhibitors (COX-2 inhibitors and COXIBs)}

Selective COX-2 inhibitors (meloxicam, lornoxicam) and the COXIBs (celecoxib, etoricoxib and parecoxib) impede the production of inflammation related prostaglandins. These agents have equal efficacy compared to NSAIDS in managing pain, fever and inflammation, but show superior gastric safety in patients with a history of upper gastrointestinal bleeding. A major deterrent to the use of selective COX-2 inhibitors are their unfavorable association with adverse cardiovascular events (myocardial infarction, stroke and heart failure) which has led to the withdrawal of most of the older products. ${ }^{31}$

\section{Opioids}

Opioids act by binding to the mu, kappa, and delta receptors located in the presynaptic neuronal cell membranes to inhibit neurotransmitter release, thereby producing analgesia. Based upon their effects on the mu receptor, opioids are divided into pure agonists, agonist-antagonists, and pure antagonists. Morphine is considered the prototype opioid and is used as the standard for comparison among other opioids, although no evidence regarding its superiority in terms of efficacy or tolerability over any other mu agonists has been proven. ${ }^{32}$ Opioid therapy is indicated in more severe forms of acute pain, including cancer palliation. Their benefit in treating cancer pain emanates from the relative safety profile, multiple routes of administration, ease of titration, reliability, and predictable effectiveness in pain suppression. Opioid use in chronic noncancer pain remains controversial. ${ }^{33}$ The misuse of opioids for management of chronic pain is a major concern with regards to adverse side effects, drug dependency and opioid abuse. ${ }^{34}$

The management of chronic cancer pain usually involves the long-term administration of pure mu receptor agonists. Initially the opioid-non-opioid combination should be used at a low starting dose. ${ }^{35}$ Short-acting pure mu receptor agonists such as morphine, oxycodone, hydromorphone or oxymorphone, are the preferred agents for moderate to severe cancer pain in opioid-naïve patients. There is a lack of evidence to support superior efficacy or tolerability of any pure opioid agent over another. However, a significant intra-individual variation in the response to the different mu agonist drugs does exist Selecting an appropriate opioid drug is usually determined by the patient's prior response to a certain agent, the clinician's experience, cost, product availability, and dosage formulation. For patients with severe pain, rapid titration of the opioid dose using intravenous dosing at short intervals, may improve pain relief, whereas switching to a long-acting formulation could improve adherence. ${ }^{36}$ There is no evidence of the difference in efficacy between long-acting formulations. The oral and transdermal route is usually preferred to the intramuscular route in managing chronic cancer pain. This allows for switching to continuous intravenous (IV) or subcutaneous (SC) infusion with hydromorphone as needed. Dose individualisation is the key to optimising the outcomes of opioid therapy. ${ }^{37}$ Dose reduction of approximately $50 \%$ is recommended for older patients with low cardiac output or respiratory compromise. Due to highly variable and prolonged half-life, pethidine has the highest risk of causing accumulation and overdose compared to other opioids. ${ }^{38}$ Transdermal fentanyl may be considered in breakthrough pain, dysphagia, and if constipation becomes problematic. Short acting transmucosal fentanyl formulations are indicated on an "as needed" basis in combination with a fixed scheduled, long-acting opioid, in managing breakthrough pain. ${ }^{39,40}$ Immediate release morphine, oxycodone, hydromorphone, or oxymorphone, or one of the combination products (paracetamol plus oxycodone) can be used as rescue therapy when the pain is not adequately controlled. ${ }^{41}$ Patients presenting with liver failure should avoid codeine and meperidine, and lower initial starting doses with prolonged dosing intervals should be given. ${ }^{42}$ Hydromorphone may be preferred for patients with renal insufficiency.

Mixed agonist-antagonist opioid agents, such as pentazocine, are generally not prescribed for cancer pain management. They have similar analgesic effects compared to the strong opioids (including morphine and meperidine), but lack any anxiolytic effect and therefore produce less CNS depression. ${ }^{43}$ Some selective centrally acting mu receptor agonists, such as tramadol, have a low affinity for these opioid receptors, whereby they preferentially inhibit serotonin and noradrenaline reuptake in addition. The synergistic action of these multiple mechanisms is responsible for the analgesic effect. Tramadol is of value in treating several pain conditions where the use of strong opioids is not required. ${ }^{44}$

All opioids may suppress respiration, cause constipation, urinary retention, nausea, vomiting, cough suppression, drowsiness and impair motor function. Naloxone may be used to reverse respiratory depression and concurrent administration of antiemetics to control nausea and vomiting. Tolerance to analgesic efficacy may develop with repeated and prolonged use. Toxicity is aggravated by alcohol or other CNS depressants. 
Opioids should be used with caution in hypotensive states and in those with impaired hepatic function..$^{42,45}$

\section{MOR-NRI ( $\mu$-opioid receptor agonists and noradrenaline reuptake inhibitors)}

Novel drugs such as tapentadol (Palexia ${ }^{\oplus}$ ) have recently been shown to produce analgesic effects comparable to that of high potency opioids including oxycodone and morphine. Tapentadol has a dual acting mechanism whereby it acts as a $\mu$-opioid receptor (MOR) agonist and noradrenaline reuptake inhibitor (NRI) to produce an analgesic effect in a synergistic manner. This new class of centrally acting agents is effective in managing both acute and chronic pain from nociceptive, inflammatory and neuropathic origins. It has a more favourable gastrointestinal tolerability and less potential for abuse compared to the opioids. ${ }^{46}$

\section{Anticonvulsants}

Antiepileptic agents are often used in the management of neuropathic pain. Carbamazepine acts as a $\mathrm{Na}+$ channel blocker in the spinal cord and higher cortical centres, thereby preventing repeated neuronal discharge. Carbamazepine (or oxcarbazepine) is indicated as specific first line therapy in the management of trigeminal neuralgia. ${ }^{25}$ Gabapentin and pregabalin bind to the voltage-gated calcium channels at the alpha 2-delta subunit and inhibit neurotransmitter release from the nerve terminals. These agents are mainly used in patients with postherpetic neuralgia, painful diabetic neuropathy, central neuropathic pain, fibromyalgia, HIV neuropathy, Guillain-Barre syndrome, chronic lumbar radicular pain, and post amputation pain syndromes. ${ }^{47}$ Other antiepileptic drugs, including topiramate, lamotrigine, levetiracetam, phenytoin, sodium valproate and clonazepam, should only be considered as second-line treatment since evidence regarding their efficacy is lacking. ${ }^{25}$

\section{Antidepressants}

Both tricyclic antidepressants (TCAs) and serotonin noradrenaline reuptake inhibitors (SNRIs) have analgesic properties which are useful in treating neuropathic pain. ${ }^{48}$ Duloxetine is effective in the treatment of painful diabetic neuropathy, fibromyalgia, chronic lower back pain and osteoarthritis. TCAs and venlafaxine prescribed for chronic pain have typically been given at lower doses than those used in depression. Both should be used with caution in patients with cardiac disease or gastrointestinal dysfunction.

\section{Glucocorticoids}

Glucocorticoids are beneficial in a multitude of pain syndromes. These include neuropathic pain, bone pain, pain associated with duct and bowel obstruction, lymphedema and cauda equina syndrome. ${ }^{49}$ Dexamethasone is usually preferred for the management of cancer-related pain, because of its long half-life and relatively low mineralocorticoid effects. Due to the longterm toxicity risks and side effects, glucocorticosteroids should only be used for the shortest time possible.

\section{NMDA receptor antagonists}

Ketamine as an N-methyl-D-aspartate (NMDA) receptor antagonist is useful for managing severe non-cancer related refractory pain in subanaesthetic doses. ${ }^{50}$ To reduce the psychotomimetic effects of ketamine, co-administration of lorazepam or haloperidol is recommended with gradual dose titration. Other NMDA antagonists, such as memantine, amantadine, or dextromethorphan may be contemplated in cancer-related neuropathic pain in patients who have not responded to conventional therapy. ${ }^{51}$

\section{GABA receptor inhibitors and agonists}

Clonazepam is the only benzodiazepine that is used for neuropathic pain. ${ }^{52}$ Clonazepam potentates GABA inhibitory mechanisms. Baclofen, a selective $G_{A B A}$ agonist, has efficacy in trigeminal neuralgia and neuropathic pain including cancer pain. ${ }^{53}$

\section{Alpha-2 adrenergic agonists}

Although the analgesic mechanism produced by alpha-2 adrenergic agonists remains unknown, it is presumed to be attributed to increased activity in monoamine-dependent endogenous pain modulating pathways in the brain and spinal cord. Spinally administered clonidine has analgesic properties in patients with cancer pain and is more efficacious for neuropathic than nociceptive pain. There is no evidence that oral clonidine is effective for chronic pain. The parenteral alpha-2 agonist, dexmedetomidine, is only used postoperatively. Apha-2 agonists produce somnolence, dry mouth and orthostatic hypotension..$^{54}$

\section{Botulinum toxin}

Botulinum toxin type A (a potent neurotoxin) may be effective in reducing the dose of opioids needed for analgesia in patients with severe post herpetic neuralgia. ${ }^{55}$

\section{Cannabis and cannabinoids}

The use of cannabis and cannabinoids for chronic pain remains controversial, although some evidence regarding their efficacy in treating neuropathic pain exists. ${ }^{56}$ Adverse effects include dizziness, dry mouth, nausea, fatigue, somnolence, euphoria, vomiting, disorientation, drowsiness, confusion, loss of balance, and hallucination. It remains an illicit drug for prescription purposes.

\section{Conclusion}

Training clinicians in both pharmacological and nonpharmacological pain management is of paramount importance. Holistic care models in primary care can improve pain management and patient outcomes. Medication should be used when needed, in conjunction with other treatment modalities like physical interventions (acupuncture, chiropractic manipulation, massage) and psychoeducational interventions such as cognitive-behavioural therapy, family therapy, psychotherapy, and patient education in order to meet treatment goals. 


\section{References}

1. van Hecke O, Torrance N, Smith BH. Chronic pain epidemiology and its clinical relevance. Br J Anaesth. 2013;111(1):13-18.

2. Elliott AM, Smith BH, Penny KI, Smith WC, Chambers WA. The epidemiology of chronic pain in the community. Lancet. 1999;354(9186):1248-52.

3. Merskey $\mathrm{H}$, Bogduk $\mathrm{N}$. Task force on taxonomy of the international association for the study of pain. Classification of chronic pain: descriptions of chronic pain syndromes and definition of pain terms. 1994;2.

4. Gaskin DJ, Richard P. The economic costs of pain in the United States. J Pain. 2012;13(8):715-24.

5. Nahin RL. Estimates of pain prevalence and severity in adults: United States, 2012. J Pain. 2015;16(8):769-80.

6. Gureje O, Von Korff M, Simon GE, Gater R. Persistent pain and well-being: a World Health Organization Study in Primary Care. JAMA. 1998;280(2):147-51.

7. Rauf W-u-N, Meyer H, Marcus TS, Becker PJ. Prevalence of chronic pain in patients attending primary healthcare facilities in south-west Tshwane. S Afr Fam Pract. 2013;55(1):85-9.

8. Rauf W-uN, Meyer HP, Marcus TS, Becker PJ. The impact of chronic pain on the quality of life of patients attending primary healthcare clinics. South Afr J Anaesth Analg. 2014;20(2):122-6.

9. Igumbor EU, Puoane T, Gansky SA, Plesh O. Pain as a reason for primary care visits: Cross-sectional survey in a rural and peri-urban health clinic in the Eastern Cape, S Afr Fam Pract.2012;54(3):256-63.

10. Gray P. Acute neuropathic pain: diagnosis and treatment. Curr Opin Anaesthesiol. 2008;21(5):590-5.

11. Frank JW, Bair MJ, Becker WC, et al. Update in pain medicine for primary care providers: a narrative review, 2010-2012. Pain Med. 2014;15(3):425-31.

12. Woolf $\mathrm{CJ}$. Pain: moving from symptom control toward mechanism-specific pharmacologic management. Ann Intern Med 2004;140(6):441-51.

13. Cleeland C, Ryan K. Pain assessment: global use of the Brief Pain Inventory. Ann Acad Med Singapore. Mar 1994;23(2):129-38.

14. Dworkin RH, Turk DC, Revicki DA, et al. Development and initial validation of an expanded and revised version of the Short-form McGill Pain Questionnaire (SF-MPQ-2). Pain. 2009;144(1-2):35-42.

15. Galer BS, Jensen MP. Development and preliminary validation of a pain measure specific to neuropathic pain: the Neuropathic Pain Scale. Neurology. 1997;48(2):332-8.

16. Moayedi M, Davis KD. Theories of pain: from specificity to gate control. J Neurophysiol. 2013;109(1):5-12.

17. Mansour AR, Farmer MA, Baliki MN, Apkarian AV. Chronic pain: the role of learning and brain plasticity. Restor Neurol Neurosci. 2014;32(1):129-39.

18. Kress $\mathrm{H}-\mathrm{G}$, Aldington $\mathrm{D}$, Alon $\mathrm{E}$, et al. A holistic approach to chronic pain management that involves all stakeholders: change is needed. Curr Med Res Opin. 2015;31(9):1743-54.

19. Park HJ, Moon DE. Pharmacologic management of chronic pain. Korean J Pain. 2010;23(2):99-108.

20. Miller $E$. The world health organization analgesic ladder. J Midwifery Womens Health. 2004;49(6):542-5.

21. Turk DC, Wilson HD, Cahana A. Treatment of chronic non-cancer pain. Lancet. 2011;377(9784):2226-35.

22. Gilron I, Baron R, Jensen T. Neuropathic pain: principles of diagnosis and treatment. Mayo Clin Proc. 2015;90(4):532-45.

23. McNicol ED, Midbari A, Eisenberg E. Opioids for neuropathic pain. Cochrane Database Syst Rev. 2013(8):Cd006146.

24. Attal N, Cruccu G, Baron R, et al. EFNS guidelines on the pharmacological treatment of neuropathic pain: 2010 revision. Eur J Neurol. 2010;17(9):1113-e1188.

25. Wiffen PJ, Derry S, Moore RA, McQuay HJ. Carbamazepine for acute and chronic pain in adults. Cochrane Database Syst Rev. 2011(1):Cd005451.

26. Bjordal JM, Ljunggren $A E$, Klovning $A$, Slordal L. Non-steroidal anti-inflammatory drugs, including cyclo-oxygenase-2 inhibitors, in osteoarthritic knee pain: meta-analysis of randomised placebo controlled trials. BMJ. 2004;329(7478):1317.

27. Trelle $S$, Reichenbach $S$, Wandel $S$, et al. Cardiovascular safety of non-steroidal anti-inflammatory drugs: network meta-analysis. BMJ. 2011;342:c7086.

28. Haag MD, Bos MJ, Hofman A, et al. Cyclooxygenase selectivity of nonsteroidal anti-inflammatory drugs and risk of stroke. Arch Intern Med. 2008;168(11):1219-24.
29. Bhala N, Emberson J, Merhi A, et al. Vascular and upper gastrointestinal effects of non-steroidal anti-inflammatory drugs: meta-analyses of individual participant data from randomised trials. Lancet. 2013;382(9894):769-79.

30. Arfè A, Scotti L, Varas-Lorenzo C, et al. Non-steroidal anti-inflammatory drugs and risk of heart failure in four European countries: nested case-control study. BMJ. 2016;354:i4857.

31. Chan FKL, Ching JYL, Tse YK, et al. Gastrointestinal safety of celecoxib versus naproxen in patients with cardiothrombotic diseases and arthritis after upper gastrointestinal bleeding (CONCERN): an industry-independent, double-blind double-dummy, randomised trial. Lancet. 2017;389(10087):2375-82.

32. Wiffen PJ, Wee B, Moore RA. Oral morphine for cancer pain. Cochrane Database Syst Rev. 2016;4:Cd003868.

33. Quigley C. Opioids in people with cancer-related pain. BMJ Clin Evid. 2008;2008.

34. Von Korff M, Kolodny A, Deyo RA, Chou R. Long-term opioid therapy reconsidered. Ann Intern Med. 2011;155(5):325-8.

35. Corli $\mathrm{O}$, Floriani I, Roberto A, et al. Are strong opioids equally effective and safe in the treatment of chronic cancer pain? A multicenter randomized phase IV 'real life' trial on the variability of response to opioids. Ann Oncol. 2016;27(6):1107-15.

36. Chou R, Clark E, Helfand M. Comparative efficacy and safety of long-acting oral opioids for chronic non-cancer pain: a systematic review. J Pain Symptom Manage. 2003;26(5):1026-48.

37. Maltoni M, Scarpi E, Modonesi C, et al. A validation study of the WHO analgesic ladder: a two-step vs three-step strategy. Support Care Cancer. 2005;13(11):888-94

38. Chatham MS, Dodds Ashley ES, Svengsouk JS, Juba KM. Dose ratios between high dose oral morphine or equivalents and oral methadone. J Palliat Med. 2013;16(8):947-50.

39. Hadley G, Derry S, Moore RA, Wiffen PJ. Transdermal fentanyl for cancer pain Cochrane Database Syst Rev. 2013(10):Cd010270.

40. Kosugi T, Hamada S, Takigawa C, et al. A randomized, double-blind, placebo-controlled study of fentanyl buccal tablets for breakthrough pain efficacy and safety in Japanese cancer patients. J Pain Symptom Manage. 2014;47(6):990-1000.

41. Bao YJ, Hou W, Kong XY, et al. Hydromorphone for cancer pain. Cochrane Database Syst Rev. 2016;10:Cd011108.

42. Oliverio C, Malone N, Rosielle DA. Opoid use in liver failure \#260. J Palliat Med. 2012;15(12):1389-91.

43. Goldstein G. Pentazocine. Drug Alcohol Depend. 1985;14(3-4):313-23.

44. Dayer P, Desmeules J, Collart L. Pharmacology of tramadol . Drugs. 1997;53 Suppl 2:18-24.

45. de Leon J, Dinsmore L, Wedlund P. Adverse drug reactions to oxycodone and hydrocodone in CYP2D6 ultrarapid metabolizers. J Clin Psychopharmacol. 2003;23(4):420-1.

46. Tzschentke TM, Christoph T, Kogel BY. The mu-opioid receptor agonist/ noradrenaline reuptake inhibition (MOR-NRI) concept in analgesia: the case of tapentadol. CNS Drugs. 2014;28(4):319-29.

47. Moore RA, Wiffen PJ, Derry S, McQuay HJ. Gabapentin for chronic neuropathic pain and fibromyalgia in adults. Cochrane Database Syst Rev. 2011(3):Cd007938.

48. Saarto T, Wiffen PJ. Antidepressants for neuropathic pain: a Cochrane review. J Neurol Neurosurg Psychiatry. 2010;81(12):1372-3.

49. Mercadante SL, Berchovich M, Casuccio A, Fulfaro F, Mangione S. A prospective randomized study of corticosteroids as adjuvant drugs to opioids in advanced cancer patients. Am J Hosp Palliat Care. 2007;24(1):13-9.

50. Prommer EE. Ketamine for pain: an update of uses in palliative care. J Palliat Med. 2012;15(4):474-83.

51. Chizh BA, Headley PM. NMDA antagonists and neuropathic pain--multiple drug targets and multiple uses. Curr Pharm Des. 2005;11(23):2977-94.

52. Nielsen $\mathrm{S}$, Lintzeris $\mathrm{N}$, Bruno $\mathrm{R}$, et al. Benzodiazepine use among chronic pain patients prescribed opioids: associations with pain, physical and mental health and health service utilization. Pain Med. 2015;16(2):356-66.

53. Yomiya K, Matsuo N, Tomiyasu $\mathrm{S}$, et al. Baclofen as an adjuvant analgesic for cancer pain. Am J Hosp Palliat Care. 2009;26(2):112-8.

54. Prommer E. Review article: dexmedetomidine: does it have potential in palliative medicine? Am J Hosp Palliat Care. 2011;28(4):276-83.

55. Xiao L, Mackey S, Hui $H$, et al. Subcutaneous injection of botulinum toxin a is beneficial in postherpetic neuralgia. Pain Med. 2010;11(12):1827-33.

56. Whiting PF, Wolff RF, Deshpande $S$, et al. Cannabinoids for Medical Use: A Systematic Review and Meta-analysis. JAMA. 2015;313(24):2456-2473. 